\title{
Optimum Transmission through the Gaussian Multiple Access Channel
}

\author{
Daniel Calabuig \\ Institute of Telecommunications and Multimedia Applications \\ Universidad Politécnica de Valencia \\ Valencia, Spain \\ Email: dacaso@iteam.upv.es
}

\author{
Ramy Gohary and Halim Yanikomeroglu \\ Department of Systems and Computer Engineering \\ Carleton University \\ Ottawa, Canada \\ Email: \{gohary, halim\}@ sce.carleton.ca
}

\begin{abstract}
In this paper we study the optimality of particular points in the capacity region of Gaussian multiple access channels (GMACs) with various power constraints. The points of interest maximize general rate objectives that arise in practical communication scenarios. Achieving these points constitutes the task of jointly optimizing time-sharing parameters, input covariance matrices and the order of decoding used by the successive interference cancellation receiver. To approach this problem Carathéodory's theorem is invoked to represent time-sharing and decoding orders jointly as a finite-dimensional matrix variable. This variable enables us to use variational inequalities to extend results pertaining to problems with linear objectives to more general, potentially nonconvex, problems. In particular, it is shown that for arbitrary objectives, if the power constraints are convex, it suffices for each user to use only one covariance matrix in all its allocated time slots. On the other hand, for arbitrary power constraints, if the objective is linear no time-sharing is necessary. These results significantly reduce the design complexity and render optimal signalling over GMAC more amenable to implementation in various practical scenarios.
\end{abstract}

\section{INTRODUCTION}

In a Gaussian multiple access channel (GMAC) multiple users send independent signals to one destination. Such a channel model arises in uplink communications of cellular systems [1]. In addition to the relevance of the GMAC to practical communication scenarios, the analysis of this channel with a sum-power constraint is closely related to the analysis of the, usually less understood, Gaussian broadcast channel (GBC). This relationship was discovered in [2], was further investigated in [3], and was used in [4] to facilitate the evaluation of the capacity region of GBC.

The capacity region of general multiple access channels was obtained in [5] and [6]. Particularizing these results to the case of Gaussian channels, it was shown therein that corner points on the boundary of the capacity region are achieved when the signal of each user is Gaussian distributed with an appropriate covariance and the receiver uses successive interference cancellation (SIC) to decode the users' signals sequentially [7]. In SIC, the receiver decodes the signal of each user while treating the signals of the set of users interfering with it as noise. After decoding, the signal of each user is stripped off from the signal interfering with the signal of the remaining users. Other points on the boundary of the capacity region can be obtained by time-sharing, whereby each decoding order and collection of users' covariance matrices are used during a fraction of the signalling duration.

An alternate approach to communication over GMACs is the so-called rate-splitting. This technique is shown in [8] to achieve every point on the boundary of the GMAC capacity region with single-user coding and without user synchronization. Achieving particular points within the capacity region of GMAC was considered in [9] and [10]. In [9], the input covariances of all users are assumed fixed and the points of interest are those that satisfy a certain fairness criterion and lie on the sum-capacity facet of the capacity region. Two cases are considered in [10]: the case of small number of users, which gives rise to a scenario in which time-sharing is feasible, and the case of large number of users, which gives rise to a scenario in which time-sharing is infeasible. In [10], fairness is not directly addressed. However, the points to be achieved are those at which the weighted sum of the rates of a given subset of users is maximized while the rates of the remaining users are restricted to some prescribed values. A somewhat similar philosophy was applied to scenarios involving broadcast channels. For instance, in [11] the GMAC-GBC duality in [2] is used to develop an algorithm that maximizes a weighted sum of the logarithms of the users' rates. In [12], another instance of a multiuser communication system is considered. Therein, the investigated system represents an interference channel and the goal is to determine the decoding order that achieves the optimal max-min fairness.

Following previous efforts to efficiently obtain particular points on the boundary of the capacity region of various multiuser systems, in this paper we focus on the GMAC scenario. In particular, we consider the joint optimization of the set of input covariance matrices, time-sharing parameters and user orderings that maximize various rate objectives with various classes of power constraints. To approach this problem, we invoke Carathéodory's theorem to represent time-sharing and decoding orders jointly as a finite-dimensional matrix variable. This variable enables us to use variational inequalities to extend results pertaining to problems with linear objectives to more general, potentially nonconvex, problems. In particular, it is shown that for arbitrary objectives, if the power constraints are convex, it suffices for each user to use only one covariance matrix in all its allocated time slots. On the 
other hand, for arbitrary power constraints, if the objective is linear no time-sharing is necessary. These results significantly reduce the design complexity and enable us to find solutions for, otherwise daunting, GMAC-based optimization problems.

\section{Preliminaries}

In this section, we provide background material necessary for subsequent development.

Lemma 1 (Variational inequalities. Necessity). Let $f: \mathcal{X} \rightarrow$ $\mathbb{R}$ be a continuously differentiable function over the convex set $\mathcal{X} \subset \mathbb{R}^{N}$. If the vector $x^{*}$ is the minimum of $f$ in $\mathcal{X}$, then

$$
\boldsymbol{x}^{* \dagger} \nabla f\left(\boldsymbol{x}^{*}\right) \leq \boldsymbol{x}^{\dagger} \nabla f\left(\boldsymbol{x}^{*}\right),
$$

for all $\boldsymbol{x} \in \mathcal{X}$, where $\nabla f(\boldsymbol{x})$ is the gradient of $f$ at $\boldsymbol{x}$.

Proof: See Appendix B of [13].

An immediate application of Lemma 1 is

Proposition 1. Let $\boldsymbol{x}^{*}$ be the optimum solution for

$$
\min _{\boldsymbol{x} \in \mathcal{X}} f(\boldsymbol{x}),
$$

where $\mathcal{X}$ is a convex set, and $f$ is continuously differentiable. Then, for the constant vector $\boldsymbol{a}=\nabla f\left(\boldsymbol{x}^{*}\right), \boldsymbol{x}^{*}$ is optimum for

$$
\min _{x \in \mathcal{X}} x^{\dagger} a \text {. }
$$

Proof: From Lemma 1, for all $x \in \mathcal{X}$, we have that $\boldsymbol{x}^{* \dagger} \boldsymbol{a} \leq \boldsymbol{x}^{\dagger} \boldsymbol{a}$. Thus, $\boldsymbol{x}^{*}$ is the minimum of $\boldsymbol{x}^{\dagger} \boldsymbol{a}$ in $\mathcal{X}$.

Complementary to Lemma 1 is the following result.

Lemma 2 (Variational inequalities. Sufficiency). Let $f: \mathcal{X} \rightarrow$ $\mathbb{R}$ be a convex continuously differentiable function over the set $\mathcal{X} \subset \mathbb{R}^{N}$. If the vector $\boldsymbol{x}^{*}$ satisfies

$$
\boldsymbol{x}^{* \dagger} \nabla f\left(\boldsymbol{x}^{*}\right) \leq \boldsymbol{x}^{\dagger} \nabla f\left(\boldsymbol{x}^{*}\right)
$$

for all $\boldsymbol{x} \in \mathcal{X}$, then, $\boldsymbol{x}^{*}=\arg \min _{\boldsymbol{x} \in \mathcal{X}} f(\boldsymbol{x})$.

Proof: See Appendix C of [13].

Remark. Notice that for Lemma 1 to hold, the set $\mathcal{X}$ must be convex, but, apart from continuous differentiability, no restrictions are imposed on the function $f$. In contrast, for Lemma 2 to hold, the function $f$ must be convex and continuously differentiable, but no restrictions are imposed on the set $\mathcal{X}$.

Lemma 2 yields a complementary result of Proposition 1.

Proposition 2. Let $\boldsymbol{x}^{*} \in \mathcal{X}$, and let $\boldsymbol{a}=\nabla f\left(\boldsymbol{x}^{*}\right)$, where $f$ is convex and continuously differentiable. If $\boldsymbol{x}^{*}=\arg \min _{\boldsymbol{x} \in \mathcal{X}} \boldsymbol{x}^{\dagger} \boldsymbol{a}$, then $x^{*}$ is also optimum for

$$
\min _{\boldsymbol{x} \in \mathcal{X}} f(\boldsymbol{x}) .
$$

Proof: The optimality assumption implies that $x^{* \dagger} \boldsymbol{a} \leq$ $\boldsymbol{x}^{\dagger} \boldsymbol{a}$ for all $\boldsymbol{x} \in \mathcal{X}$. Therefore, from Lemma $2, x^{*}$ is the minimum of $f$ in $\mathcal{X}$.

Propositions 1 and 2 will enable us to draw some relationships between solutions of general problems and solutions of problems with linear objectives. Combining these proposition for cases in which $\mathcal{X}$ is convex and $f$ is convex and continuously differentiable yields the following corollary.

Corollary 1. Let $f: \mathcal{X} \rightarrow \mathbb{R}$ be convex and continuously differentiable and let $\mathcal{X} \subset \mathbb{R}^{N}$ be convex. Then $\boldsymbol{x}^{*}=\arg \min _{\boldsymbol{x} \in \mathcal{X}} f(\boldsymbol{x})$ if and only if $\boldsymbol{x}^{*}=\arg \min _{\boldsymbol{x} \in \mathcal{X}} \boldsymbol{x}^{\dagger} \boldsymbol{a}$, where $\boldsymbol{a}=\nabla f\left(\boldsymbol{x}^{*}\right)$.

\section{SYSTEM MODEL AND OPTIMIZATION}

The GMAC is composed of $K$ users transmitting to one base station. The number of transmit antennas of the $k$-th user is denoted by $N_{k}, k=1, \ldots, K$, and the number of receive antennas at the base station is denoted by $N_{K+1}$. The received signal is given by

$$
\boldsymbol{y}=\sum_{k=1}^{K} \boldsymbol{H}_{k} \boldsymbol{x}_{k}+\boldsymbol{z},
$$

where $\boldsymbol{H}_{k} \in \mathbb{C}^{N_{K+1} \times N_{k}}$ is the channel matrix of the $k$-th user and $\boldsymbol{x}_{k} \in \mathbb{C}^{N_{k}}$ is its transmitted signal. The Gaussian noise at the base station is denoted by $z \in \mathbb{C}^{N_{K+1}}$, which is assumed to satisfy $\mathrm{E}\left[\boldsymbol{z} z^{\dagger}\right]=\boldsymbol{I}$.

The limitation of the system is the available power. Let $\boldsymbol{Q}_{k}=\mathrm{E}\left[\boldsymbol{x}_{k} \boldsymbol{x}_{k}^{\dagger}\right]$ and let $\overline{\boldsymbol{Q}}=\boldsymbol{Q}_{1} \oplus \cdots \oplus \boldsymbol{Q}_{K}$, that is, $\overline{\boldsymbol{Q}}$ is block diagonal with the matrices $Q_{1}, \cdots, Q_{K}$ along the diagonal. We consider systems with $L$ power constraints which can be expressed as $g_{l}(\operatorname{diag}(\overline{\boldsymbol{Q}})) \leq 0, l=1, \ldots, L$. Let

$$
\mathcal{P}=\left\{\overline{\boldsymbol{Q}} \mid \overline{\boldsymbol{Q}} \succeq 0, g_{l}(\operatorname{diag}(\overline{\boldsymbol{Q}})) \leq 0, l=1, \ldots, L\right\}
$$

be the set of all feasible $\bar{Q}$. For practical considerations, this set can be assumed to be bounded.

Corner points of the GMAC capacity region can be achieved by using an SIC receiver. To achieve a particular corner, the receiver orders the users and decodes their signals sequentially. Decoded signals are stripped off the signal used to decode subsequent users. In other words, the amount of interference observed in decoding the signal of a particular user depends on the ordering. Hence, to maximize a given objective, the receiver must determine the optimal user ordering. More precisely, let $\pi_{1}, \cdots, \pi_{K}$ ! be the set of all $K$ ! permutations, where $\pi_{i}(j)$ refers to the user in the $j$-th position of the $i$-th ordering. For this ordering, the $k$-th user is able to achieve:

$$
r_{k i}(\overline{\boldsymbol{Q}})=\log \frac{\left|\boldsymbol{I}+\sum_{j \geq \pi_{i}^{-1}(k)} \boldsymbol{H}_{\pi_{i}(j)} \boldsymbol{Q}_{\pi_{i}(j)} \boldsymbol{H}_{\pi_{i}(j)}^{\dagger}\right|}{\left|\boldsymbol{I}+\sum_{j>\pi_{i}^{-1}(k)} \boldsymbol{H}_{\pi_{i}(j)} \boldsymbol{Q}_{\pi_{i}(j)} \boldsymbol{H}_{\pi_{i}(j)}^{\dagger}\right|} .
$$

The GMAC capacity region is the convex hull of all rate vectors that are achievable with all orderings and all covariance matrices in $\mathcal{P}$ in (1). This implies that, in order to reach certain rate vectors, we may need to use convex combinations of $M$ rate vectors with $M$ collections of covariance matrices $\left\{\overline{\boldsymbol{Q}}^{(m)}\right\}_{m=1}^{M}$ and $M$ permutations $\left\{\pi_{i}(\cdot)\right\}$. For notational convenience, the collection of $M$ permutations will be denoted by $\nu$. Convex combinations can be implemented in practice by time-sharing using $M$ non-negative coefficients, $a_{1}, \ldots, a_{M}$, where $a_{m} \geq 0, m=1, \ldots, M$ and $\sum_{m=1}^{M} a_{m}=1$. These 
coefficients represent the percentage of time during which each of the $M$ rate vectors is used. Hence, with time-sharing included, the $k$-th user is able to achieve the following rate

$$
\sum_{m=1}^{M} a_{m} \log \frac{\left|\boldsymbol{I}+\sum_{j \geq \nu_{m}^{-1}(k)} \boldsymbol{H}_{\nu_{m}(j)} \boldsymbol{Q}_{\nu_{m}(j)}^{(m)} \boldsymbol{H}_{\nu_{m}(j)}^{\dagger}\right|}{\left|\boldsymbol{I}+\sum_{j>\nu_{m}^{-1}(k)} \boldsymbol{H}_{\nu_{m}(j)} \boldsymbol{Q}_{\nu_{m}(j)}^{(m)} \boldsymbol{H}_{\nu_{m}(j)}^{\dagger}\right|}
$$

where $\nu_{m}(\cdot)$ is the $m$-th permutation in $\boldsymbol{\nu}$, and $\boldsymbol{Q}_{k}^{(m)}$ is the covariance matrix of the signal of the $k$-th user in the $m$-th collection. Fortunately, the maximum number of rate vectors required to be combined is given by Carathéodory's theorem, which implies that it suffices to set $M=K+1$ to reach every point in the GMAC capacity region.

Being explicit in permutations, the characterization in (2) is not well-suited for finding collections of covariances, timesharing parameters and user-orderings that enable practically important objectives to be optimized. To circumvent this difficulty, the time-sharing parameters and user permutations are combined in what we refer to as the time-sharing matrix $\boldsymbol{\alpha} \in \mathbb{R}^{(K+1) \times K !}$. The $m i$-th element of $\boldsymbol{\alpha}, \alpha_{m i}$, represents the percentage of time where the $m$-th collection of covariance matrices is used with the $i$-th ordering. Hence, this representation renders the elements of $\boldsymbol{\alpha}$, not only time-sharing weights for generating convex combinations, but also indicators of the used orderings. In particular, $\alpha_{m i}=a_{m}>0$ if the permutation $\nu_{m}=\pi_{i}$ and $\alpha_{m i}=0$, otherwise. Using this notation, we express any achievable rate of the user $k$ of the form in (2) as $\sum_{m=1}^{K+1} \sum_{i=1}^{K !} \alpha_{m i} r_{k i}\left(\overline{\boldsymbol{Q}}^{(m)}\right)$. By introducing the matrix $\boldsymbol{\alpha}$, we obtained a form that is more convenient for subsequent optimization, but at the expense of increasing the dimensionality from combining $K+1$ rate vectors to combining $(K+1)$ ! ones. However, since it suffices to timeshare no more than $K+1$ rate vectors, any rate vector of the capacity region can be achieved with a time-sharing matrix with, at most, $K+1$ non zero elements. The entries of the time-sharing matrix must belong to the unit simplex

$$
\mathcal{S} \triangleq\left\{\boldsymbol{\alpha} \mid \sum_{m=1}^{K+1} \sum_{i=1}^{K !} \alpha_{m i}=1, \alpha_{m i} \geq 0, \forall m, i\right\} .
$$

Let $\mathcal{Q}=\left\{\overline{\boldsymbol{Q}}^{(m)}\right\}_{m=1}^{K+1}$. Then, the GMAC capacity region can be expressed as the union of rate vectors $\boldsymbol{\rho}(\boldsymbol{\alpha}, \mathcal{Q})$ with the $k$-th entry given by

$$
\rho_{k}(\boldsymbol{\alpha}, \mathcal{Q})=\sum_{m=1}^{K+1} \sum_{i=1}^{K !} \alpha_{m i} r_{k i}\left(\overline{\boldsymbol{Q}}^{(m)}\right), k=1, \ldots, K .
$$

With notation established, our goal now is to consider objectives $f: \mathbb{R}^{K} \rightarrow \mathbb{R}$ with relevance to practical applications and to characterize the solution of problems of the form:

$$
\min _{\boldsymbol{\alpha}, \mathcal{Q}} f(\boldsymbol{\rho}(\boldsymbol{\alpha}, \mathcal{Q}))
$$

subject to $\boldsymbol{\alpha} \in \mathcal{S}$,

$$
\overline{\boldsymbol{Q}}^{(m)} \in \mathcal{P}, m=1, \ldots, K+1 .
$$

The number of variables in this problem is $(K+1) !+(K+$ 1) $\sum_{k} N_{k}^{2}$, where the first term is the number of variables in $\boldsymbol{\alpha}$, and the second term is the number of variables in $\mathcal{Q}$. We will later present results that will enable us to significantly reduce this number.

\section{PRoblems With LiNEAR OBJECTIVES}

In this section we will derive results for problems with linear objectives. These results will be used in the following section with Propositions 1 and 2 and Corollary 1 to draw valuable insight into problems with nonlinear objectives.

When the objective is linear, (3) can be cast as

$$
\max _{\boldsymbol{\alpha}, \mathcal{Q}} \sum_{k=1}^{K} w_{k} \rho_{k}(\boldsymbol{\alpha}, \mathcal{Q}),
$$

subject to (3b) and (3c),

where $\left\{w_{k}\right\}_{k=1}^{K}$ are constant, not necessarily positive, weights. The purpose for considering potentially non-positive weights will become clear when we discuss nonlinear objectives.

With $\left\{w_{k}\right\}_{k=1}^{K}$ given, the optimum user ordering is known and is given in the following lemma.

Lemma 3. For problems with linear objectives and arbitrary power constraints, the optimum decoding order is the increasing order of weights, that is, if $w_{1} \leq \cdots \leq w_{K}$, it is optimal to decode user 1 first, followed by user 2, and so on.

Proof: See Appendix E of [13] for details, and a similar proof in [14].

Remark. 1) Lemma 3 implies that if maximizing the (linear) objective requires time-sharing of multiple rate vectors, each of these vectors can be achieved with the same decoding order.

2) When the weights are distinct, the decoding order is unique, unless the received signals of a subset of users are either zero or orthogonal to each other. The uniqueness proof is omitted for brevity. These cases result in trivial consequences and will be ignored throughout.

3) For multiple equal weights, the decoding of the respective users can be interchanged without loss of optimality.

From Lemma 3, the optimization can focus on just one ordering, whereupon the number of variables is reduced to $(K+1)+(K+1) \sum_{k} N_{k}^{2}$.

To complete the characterization of the optimal solution of (4) we have the following result.

Lemma 4. For problems with linear objectives and arbitrary power constraints, the objective is maximized with one collection of covariance matrices.

Proof: The proof begins by assuming that the optimum is reached using two collections of covariance matrices, and proceeds to show that one of these collections suffices to reach the optimum. See Appendix F of [13].

Combining Lemmas 3 and 4 , it can be seen that for problems with linear objectives and arbitrary power constraints, 
time-sharing is not necessary to solve (4). In addition, the number of variables is now reduced to just $\sum_{k} N_{k}^{2}$. In Section $\mathrm{V}$ these lemmas will be used to facilitate the solution of a wide class of problems with nonlinear objectives.

\section{PROBlEMS WITH NONLINEAR OBJECTIVES}

We will now use the preliminaries in Section II and the results of Section IV to gain insight into problems with general objectives. Moreover, in Section V-A, we will consider a class of problems for which the optimization can be significantly simplified. All these results are applied to an example in Section V-B. We begin by recalling that our notation implies that the $m i$-th entry of the optimal time-sharing matrix is nonzero if and only if the $i$-th decoding order, $\pi_{i}(\cdot)$, and the $m$-th collection of the covariance matrices, $\overline{\boldsymbol{Q}}^{(m)}$, are optimal. Next, we present our first result of this section.

Proposition 3. Let $\boldsymbol{\alpha}^{*}$ and $\mathcal{Q}^{*}$ be optimum for the general problem in (3). Let $w_{0}=0$ and $\left\{w_{k}\right\}_{k=1}^{K}$ be given by

$$
w_{k}=-\left.\frac{\partial f(\boldsymbol{x})}{\partial x_{k}}\right|_{\boldsymbol{x}=\boldsymbol{\rho}\left(\boldsymbol{\alpha}^{*}, \mathcal{Q}^{*}\right)} \quad k=1, \ldots, K .
$$

Let the users be labelled so that $w_{1} \leq \cdots \leq w_{K}$. Then, for each strictly positive $\alpha_{m i}^{*} \in \boldsymbol{\alpha}^{*}$

1) $\pi_{i}(\cdot)$ is ordered as $w_{1}, \ldots, w_{K}$ (multiple values of $i$ will satisfy this condition if multiple weights are equal), and

2) the collection $\overline{\boldsymbol{Q}}^{*(m)}$ solves

$$
\max _{\overline{\boldsymbol{Q}}} \sum_{k=1}^{K}\left(w_{k}-w_{k-1}\right) \log \left|\boldsymbol{I}+\sum_{j \geq k} \boldsymbol{H}_{j} \boldsymbol{Q}_{j} \boldsymbol{H}_{j}^{\dagger}\right|,
$$

subject to $\bar{Q} \in \mathcal{P}$.

Proof: The proof hinges on the convexity of the GMAC capacity region. See Appendix G of [13] for details.

Apart from implicit differentiability, no other assumptions on $f$ are imposed in deriving the necessary conditions of Proposition 3. However, if the objective $f$ is also convex, the conditions of Proposition 3 are also sufficient for optimality. This statement is formalized in the next theorem.

Theorem 1. For the optimization problem (3), if the objective $f$ is convex and continuously differentiable, then the timesharing matrix $\alpha^{*}$ and the collection of covariance matrices in $\mathcal{Q}^{*}$ are optimum if and only if they satisfy conditions 1 and 2 of Proposition 3.

Proof: The proof of necessity follows from Proposition 3, whereas the proof of sufficiency follows from applying Proposition 2. See Appendix H of [13] for details.

\section{A. Convex and nondecreasing power constraints}

We now restrict our attention to a class of problems in which the power constraints, $g_{l}$ in $(1), l=1, \ldots, L$, are convex and nondecreasing in each component. It is shown that, for this class of problems, any rate vector in the capacity region can be achieved using only one collection of covariance matrices.
In some sense, this result generalizes Lemma 4, particularly when power constraints are convex and nondecreasing. The main result of this section is given in the following theorem.

Theorem 2. For the optimization problem in (3), let the power constraint functions, $g_{l}, l=1, \ldots, L$ that generate the feasible set $\mathcal{P}$ in (1) be convex and nondecreasing in each component. Then, any achievable rate vector in the corresponding GMAC can be achieved with one collection of covariance matrices.

Proof: To prove this theorem, we assume that a feasible time-sharing matrix and a feasible collection of covariance matrices are given. Using these, a certain collection of covariance matrices is synthesized and is shown to achieve higher rates than the given matrices. See details in Appendix I of [13].

When $g_{l}, l=1, \ldots, L$ are convex and nondecreasing, Theorem 2 significantly reduces the complexity of solving and analyzing the problem in (3). In particular, in such cases, the time sharing matrix $\alpha$ can be replaced with a time-sharing vector $\beta \in \mathbb{R}^{K}$, where $\beta$ lies in the unit $K$ !-dimensional simplex, $\mathcal{S}=\left\{\boldsymbol{\beta} \mid \boldsymbol{\beta} \geq 0, \sum_{i} \beta_{i}=1\right\}$. Using this notation, achievable rate vectors can be expressed as $\rho(\beta, \bar{Q})$, where

$$
\rho_{k}(\boldsymbol{\beta}, \overline{\boldsymbol{Q}})=\sum_{i=1}^{K !} \beta_{i} r_{k i}(\overline{\boldsymbol{Q}}), \quad k=1, \ldots, K
$$

Using this result, the number of variables is reduced to $K !+\sum_{k} N_{k}^{2}$, which is significantly less than the number of variables in (3). For simplicity, this formulation will be used in the next section.

\section{B. Algorithm and Application}

1) Algorithm: In this section, we develop an algorithm that uses the necessary and sufficient conditions of Theorem 1 to solve a class of nonconvex GMAC optimization problems that might, otherwise, be considered intractable. To apply Theorem 1 , the objective must be convex in $\left\{\rho_{k}(\beta, \bar{Q})\right\}_{k=1}^{K}$, but not necessarily convex in the input covariance matrices or the time-sharing parameters. The power constraints are assumed to be convex nondecreasing functions of the covariance matrices. With the step-size sufficiently small, the developed algorithm is guaranteed to converge to the optimal solution. The main steps of the algorithm are given below. Assuming that an initial time-sharing vector and collection of covariances are given, at each iteration $t$, the algorithm

1) uses (5) to compute a vector of weights;

2) searches for the optimum covariance matrices that solves the problem with a linear objective corresponding to these weights; and

3) with the obtained covariance matrices fixed, searches for the time-sharing vector that optimizes the objective of the original problem.

Algorithm details and convergence analysis are provided in Appendix K of [13].

2) Application: We now provide an instance in which the developed algorithm is used to solve a two-user GMAC optimization problem with a nonconvex objective and convex 


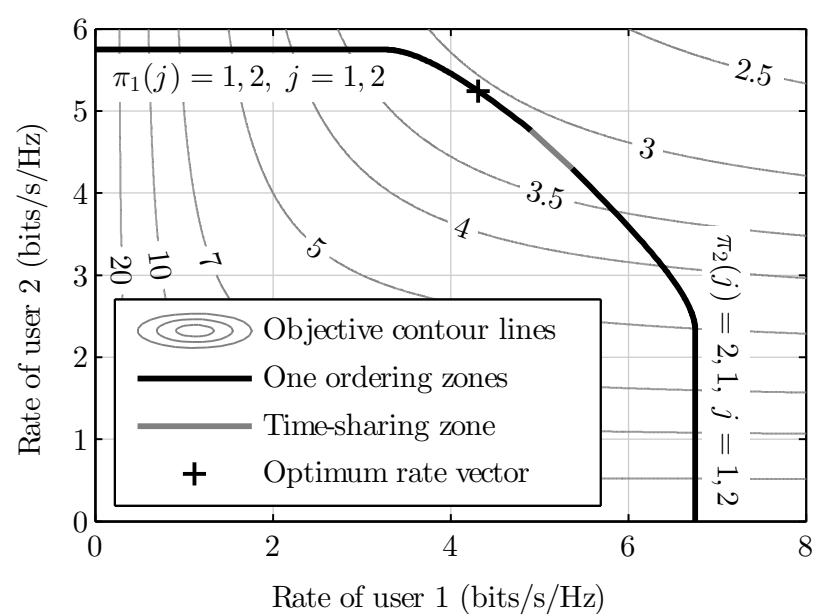

Fig. 1. The time-sharing section is highlighted with grey color.

nondecreasing power constraints. In this instance, the objective is to minimize the total completion time [15], that is, the time required to transmit the data stored in the buffers of the two users. Each user has two transmit antennas and a power budget of $P=10 \mathrm{~dB}$. The destination has two receive antennas and the channel matrices are given by

$$
\begin{gathered}
\boldsymbol{H}_{1}=\left(\begin{array}{cc}
0.32 & -0.06 \\
-0.72 & -0.88
\end{array}\right)+\sqrt{-1}\left(\begin{array}{cc}
-0.15 & -1.38 \\
-1.34 & -0.01
\end{array}\right), \\
\boldsymbol{H}_{2}=\left(\begin{array}{ll}
-0.21 & 0.29 \\
-0.08 & 0.91
\end{array}\right)+\sqrt{-1}\left(\begin{array}{cc}
-0.65 & 0.15 \\
0.13 & 1.39
\end{array}\right) .
\end{gathered}
$$

Using these matrices and assuming that the users have independent power constraints, the GMAC capacity region can be readily obtained; cf. Figure 1 . Let $b_{1}=5$ and $b_{2}=10$ represent the amount of data stored in the buffers of user 1 and 2, respectively. In this case, the optimization problem that yields the smallest completion time can be expressed as

$$
\begin{aligned}
\min _{\boldsymbol{\beta}, \overline{\boldsymbol{Q}}} & \frac{b_{1}}{\rho_{1}(\boldsymbol{\beta}, \overline{\boldsymbol{Q}})}+\frac{b_{2}}{\rho_{2}(\boldsymbol{\beta}, \overline{\boldsymbol{Q}})}, \\
\text { subject to } & \beta_{1}+\beta_{2}=1, \beta_{i} \geq 0, i=1,2, \\
& \boldsymbol{Q}_{k} \succeq 0, \operatorname{tr}\left(\boldsymbol{Q}_{k}\right) \leq P, k=1,2 .
\end{aligned}
$$

The contour lines of this objective are depicted in Figure 1. The optimum rate vector is marked by the symbol ' + '. The time-sharing weights, the decoding order, and covariance matrices required to achieve this vector are generated by the algorithm developed in Section V-B1.

\section{CONCLUSION}

We considered a GMAC scenario in which the goal is to maximize a general non-linear objective, provided that multiple power constraints are satisfied. The variables that underlie this optimization are the input covariance matrices of the users, their time-sharing parameters and their decoding order. As such, the considered problems fall under the category of mixed-integer optimization problems, which are generally difficult to solve. To circumvent this difficulty, we invoked Carathéodory's theorem and variational inequalities to analyze problems with general possibly non-convex objectives. This analysis enabled us to derive: 1) necessary optimality conditions for general problems; and 2) necessary and sufficient optimality conditions for problems with objectives that are convex in the rates, but not in the optimization variables. Drawing insight into these conditions, we designed an algorithm for solving a broad class of practical, but generally difficult to solve, GMAC optimization problems. We suspect that, using the GMAC-GBC duality, our results can be utilized to determine the optimal transmission parameters of the dirtypaper coding scheme.

\section{ACKNOWLEDGMENTS}

The authors would like to thank Dr. Melda Yuksel, TOBB University of Economics and Technology, Turkey, for her valuable comments.

The work of the first author was supported by a Marie Curie International Outgoing Fellowship (IOF) of the European Commission under the project COMIC (253990). The work of the second and third authors was supported in part by Huawei Technologies Canada Co., Ltd. and in part by NSERC Canada.

\section{REFERENCES}

[1] T. M. Cover and J. A. Thomas, Elements of Information Theory. New York: Wiley, 1991.

[2] S. Vishwanath, N. Jindal, and A. Goldsmith, "Duality, achievable rates, and sum-rate capacity of Gaussian MIMO broadcast channels," IEEE Trans. Inf. Theory, vol. 49, pp. 265-2668, Oct. 2003.

[3] W. Yu, "Uplink-downlink duality via minimax duality," IEEE Trans. Inf. Theory, vol. 52, pp. 361-374, Feb. 2006.

[4] H. Weingarten, Y. Steinberg, and S. Shamai (Shitz), "The capacity region of the Gaussian multiple-input multiple-output broadcast channel," IEEE Trans. Inf. Theory, vol. 52, pp. 3936-3964, Sept. 2006.

[5] R. Ahlswede, "Multi-way communication channels," in Proc. 2nd Int. Symp. Information Theory, (Tsahkadsor, Armenia, USSR), pp. 103-105, Sept. 1971.

[6] H. Liao, Multiple access channels. PhD thesis, Univ. Hawaii, 1972.

[7] A. D. Wyner, "Recent results in the shannon theory," IEEE Trans. Inf. Theory, vol. 20, pp. 2-10, Jan. 1974.

[8] B. Rimoldi and R. Urbanke, "A rate-splitting approach to the Gaussian multiple-access channel," IEEE Trans. Inf. Theory, vol. 42, pp. 364-375, Mar. 1996.

[9] M. A. Maddah-Ali, A. Mobasher, and A. Khandani, "Fairness in multiuser systems with polymatroid capacity region," IEEE Trans. Inf. Theory, vol. 55, pp. 2128-2138, May 2009.

[10] W. Mesbah and H. Alnuweiri, "Joint rate, power, and decoding order optimization of multiuser MIMO systems," IEEE Trans. Wireless Commun., vol. 10, pp. 1681-1686, June 2011.

[11] J. Liu and Y. T. Hou, "Weighted proportional fairness capacity of Gaussian MIMO broadcast channels," in Proc. IEEE Int. Conf. Comp. Commun. (INFOCOM), (Phoenix), pp. 1058-1066, Apr. 2008

[12] M. A. Maddah-Ali, H. Doost, and A. Khandani, "Optimal order of decoding for max-min fairness in $k$-user memoryless interference channels," in Proc. IEEE Int. Symp. Inf. Theory, (Nice), pp. 2866-2870, June 2007.

[13] D. Calabuig, R. Gohary, and H. Yanikomeroglu, "Optimum transmission through the gaussian multiple access channel." http://dacaso.webs.upv. es/hidden/User_Ordering.pdf, Jan. 2013.

[14] D. N. C. Tse and S. V. Hanly, "Multiaccess fading channels-part i: Polymatroid structure, optimal resource allocation and throughput capacities," IEEE Trans. Inf. Theory, vol. 44, pp. 2796-2815, Nov. 1998.

[15] Y. Liu and E. Erkip, "Completion time in multi-access channel: An information theoretic perspective," in Proc. IEEE Inform. Theory Wkshp. (ITW), (Paraty, Brazil), pp. 708-712, Oct. 2011. 